\title{
Didaktik der Geographie Wissenschaft aus eigenem Recht oder Anhängsel der Geographie?
}

\section{Einleitende Gedanken zum folgenden Artikel von Helmuth Köck}

Der von Prof. Köck eingesandte und im Rahmen eines Geographischen Kolloquiums vorgetragene und diskutierte Aufsatz könnte auch unter Schweizer Geographinnen und Geographen als Anregung für eine weitere Diskussion dienen. Zusammen mit dem vor einem Jahr erschienen Themenheft «Didaktik der Geographie» (Geographica Helvetica, Heft 1, 1989) bildet der Artikel von Köck eine interessante Grundlage, um auch in der Schweizer Geographie der Frage «Ist die Geographiedidaktik eine Wissenschaft aus eigenem Recht oder ein Anhängsel der Geographie?» nachzugehen.

Für H. Köck ist die Geographiedidaktik eine Wissenschaft aus eigenem Recht, was er wissenschaftstheoretisch, wissenschaftssystematisch, wissenschaftsorganisatorisch und wissenschaftsgeschichtlich begründet. Geographiedidaktik bedeutet für ihn institutionalisiertes Lehren und Lernen von Aussagen über die räumliche Ordnung der Geosphäre und hat nicht wie die Geographie die räumliche Ordnung der Geosphäre zum Gegenstand.

Geographiedidaktik ist daher keine Subdisziplin der Geographie, sondern aus begriffslogischer und wissenschaftssystematischer Sicht eher eine Subdisziplin der Didaktik. Disziplingeschichtlich ist die Geographiedidaktik noch sehr jung (akademische Disziplin in der BRD etwa seit 1960). Nach Köck wurden geographiedidaktische Fragen aber auch schon in vorakademischer Zeit unabhängig von der Geographie, aus dem didaktisch-methodischen Denken allgemein (vgl. Comenius, Gutsmuths) abgeleitet. Da es sich um eine relativ junge Disziplin handelt, sollte ihr Entwicklungsnotwendigkeit und Entwicklungsfähigkeit zugestanden werden.

Besonders interessant für eine Diskussion sind die Ausführungen zur wissenschaftsorganisatorischen Stellung der Geographiedidaktik. Die Stellung der Geographiedidaktik, z. B. als integriertes Fach an einem Geographischen Institut, führt dazu, daß sie in der Forschung meist ein Schattendasein führt. Vorurteile und eine Geringschätzung vieler Geographen gegenüber der Geographiedidaktik führen oft zu einem Profilierungszwang und zwar außerhalb der Didaktik, nämlich in der allgemeinen geographischen Forschung.
Die Stellung der Geographiedidaktik an den Schweizer Universitäten ist verschieden von derjenigen in der BRD. Auch innerhalb der Institute bestehen große Unterschiede. Die Geographiedidaktik bewegt sich hauptsächlich im Rahmen der Geographielehrerausbildung. Als Fachdidaktiker werden in der Regel Mittelschullehrer gewählt. Ihre Integration an den einzelnen Instituten ist unterschiedlich. Außer am Geographischen Institut der Universität Bern besteht in der Schweiz kein Lehrstuhl für Fachdidaktik der Geographie. Theoriedidaktische Forschung ist in der Schweiz daher nur schwer möglich. Vor allem beim «Berner Modell» (AERNI/HASLER 1989:38ff.) werden praktische geographische Beiträge wie etwa Lehrmittel, Dokumentationsmaterial, Exkursionen usw. speziell für die Lehreraus- und fortbildung angeboten. An anderen Instituten, wie etwa in Zürich, ist ein diesbezügliches Angebot kaum vorhanden.

Der Verein Schweizerischer Geographielehrer (VSGg) setzt sich zurzeit mit der Neuformulierung der Rahmenlehrpläne für das Fach Geographie auseinander. Er sucht Antworten auf die Frage, welche Beiträge die Geographie zur Bildung und Ausbildung künftiger Maturanden liefern könnte und müßte (TSCHANZ 1989:53ff.).

Unabhängig von der gegenwärtigen Stellung der Geographiedidaktik an den Schweizer Hochschulen, stellt sich auch die Frage der zeitlichen Dringlichkeit, mit welcher ihre Bedeutung diskutiert werden sollte. Diese wird auch im «Newsletter» (No. 19) der "Commission Geographical Education» der IGU betont. In «GeoJournal» zum Thema «Geography and General Education» kommt TIETZE (1990) zu folgenden Schlußfolgerungen und einem Aufruf: "The comprehensive nature of geography is being lost, while at the same time-and this is the dilemma-there is an increasingly important necessity to understand and to effectively manage the home of mankind: the Earth. Mistakes made in the context of man-land (man-water; man-air) relationship are inevitably lead-

Eva Buff Keller, Dr., Geographisches Institut ETH, Winterthurerstrasse 190, 8057 Zürich. 
ing to ever more serious, often irreversible catastrophes (...) There is only one logical response: Immediately there must be an improvement in the level of general education in geography along with much better interlinking of geo- and biosciences. Without such improvement, the risk of continued environmental degradation may soon reach fatal dimensions. Relative geo-illiteracy is dangerous (...) The International Geographical Union, represented by its Commissions and Study Groups, must take initiatives and identify goals and approaches to enhancing public awareness of geo-literacy. The IGU is advised to stimulate meetings and publications which are adequately compelling to rock the sleepy public to action. The entire community of geographers all over the world is requested to act accordingly to meet this responsibility."

Damit die drängenden Probleme dieser Geosphäre und der in ihr lebenden Menschheit vielleicht doch noch gelöst werden können, ist eine intensive Förderung der geographischen Erziehung und damit der Geographiedidaktik, welche optimales Lehren und Lernen von geographischen Inhalten erforscht und lehrt, nötig.

Zum Schluß möchte ich noch einige Fragen und Thesen zu diesem Thema aufwerfen:

- Warum führt die Geographiedidaktik auch an manchen Schweizer Instituten ein Schattendasein?

- Auf dem Hintergrund der zunehmend komplexeren und gefährlicheren Umweltprobleme sollte die Rolle der Geographiedidaktik neu überdacht werden. Wird sie stärker gewichtet, so könnte der Transfer geographischer Forschung und Praxis für eine möglichst breite Bevölkerungsschicht noch besser stattfinden.

- Interessant wäre auch die Frage, wie Frauen die von Köck beschriebene Image- und Profilierungsproblematik erleben. Meine Annahme geht dahin, daß Frauen diese Problematik nicht oder weniger erleben. Es könnte sein, daß Frauen, aufgrund ihrer Sozialisation, der Tätigkeit des Leh- rens und Lernens und der damit verbundenen Beziehungsebene mehr Bedeutung beimessen. Um diese Vermutung beweisen zu können, müßten, neben entsprechenden Untersuchungen zu dieser Thematik, auch vermehrt Frauen im universitären Lehrkörper an den entsprechenden Stellen vertreten sein.

Die Zeit drängt. Lösungen für die vielfältigen Probleme sind dringend gesucht.

Wäre es daher nicht wichtig, auch an Schweizer Hochschulen vermehrt Geographie- und Fachdidaktische Institute zu schaffen, die sowohl in Lehre als auch in Forschung volle Entfaltungsmöglichkeiten haben? Nur so würde erreicht, daß Ergebnisse aus Forschung und Praxis optimal vermittelt und verantwortungsbewußtes Handeln bewirkt werden könnte.

Ich bin daher der Meinung, daß über die Frage der Bedeutung und des Stellenwertes einer Didaktik der Geographie als dringendes Gebot der Zeit von Geographinnen und Geographen in- und außerhalb der Hochschule intensiv nachgedacht werden müsste.

Ich könnte mir vorstellen, daß zu diesem Themenkreis eine Diskussion entsteht, aus der vielleicht auch einzelne Stellungnahmen in einem weiteren Heft der Geographica Helvetica abgedruckt werden können.

\section{Literaturverzeichnis}

AERNI, K. und HASLER, M. (1989): Das «Berner Modell» Geographielehrerausbildung und -fortbildung am Geographischen Institut der Universität Bern. In: Geograhica Helvetica 44/1989, 38-43.

TIETZE, W. (1990): Conclusion and Appeal. Quotation out of GeoJournal/Jan. 1990. In: Newsletter No. 19, IGU, Commission Geographical Education, Dez. 1989.

TSCHANZ, M. (1989): Bildungsziele des Geographieunterrichts im Jahre 2000. In: Geographica Helvetica 44/1989, 53-55. 